\title{
Progressive Histiocytosis of Non-Epitheliotropic Dendritic Cells in a Feline
}

\author{
Nayadjala Távita Alves dos Santos, Jássia da Silva Meneses, João Batista Machado Alves Neto, \\ Thaís Ribeiro Félix, José de Jesus Cavalcante dos Santos, Rômulo Freitas Francelino Dias, \\ Luiz Eduardo Carvalho Buquera \& Ricardo Barbosa Lucena
}

\begin{abstract}
Background: Histiocytic tumors in felines are nodules that commonly develop on limbs and head extremities. They can be divided into many subtypes including cutaneous histiocytoma, histiocytic sarcoma, reactive fibrohistiocytic nodule, Langerhans cell histiocytosis, and progressive feline dendritic cell. Despite the same origin, they have behaviors that differ from each other, thus it is important to confirm diagnosis with histopathological and immunohistochemical tests, because early identification can facilitate prognosis and treatment. In this study, we describe the pathological and immunohistochemical characteristics, enabling differentiation feline neoplasms derived from histiocytes.

Case: A 5-year-old, crossbreed, male, feline presented with a nodulation at the base of the left ear. The mass was slow growing, partially alopecic, with no other changes associated with tumor development. The nodule was round and circumscribed, movable, with an elevated surface. He was referred for surgery and an elliptical sample around the tumor was carefully dissected. Routine histopathological evaluation was performed with hematoxylin and eosin (HE), as well as immunohistochemistry. Histopathology showed circumscribed proliferation of histiocytic cells, with abundant and eosinophilic cytoplasm. The proliferative cells were large and rounded, extending from the superficial dermis and basement membrane to the deep dermis. At the extremities, some cells had visible vacuoles. Mitotic activity ranged from 3 to 4 mitoses per field in 40x magnification. Immunohistochemistry showed positive staining for histocompatibility complex MCII and lysozyme antibodies, marking histiocytic cells. Labeling was positive for CD20 in cells of lymphoid lineage B and negative for E-cadherin. Histiocytic cells did not invade the epidermis; hence, proliferation was classified as nonepitheliotropic. These methods contribute to the literature regarding the diagnosis of this rare tumor. Therefore, histological as well as immunohistochemical evaluation are important bfor confirming clinical diagnosis of histiocytic proliferation non-epitheliotropic.

Discussion: Progressive histiocytosis of feline dendritic cells, in both epitheliotropic and non-epitheliotropic forms, is considered a clinically progressive and rare disorder. There are reports which include cytological, clinical, histological and immunohistochemical examinations, but the diagnostic characteristics regarding the non-epitheliotropic classification have not yet been properly identified. Nodulations are predominantly observed in head and limb regions, usually non-ulcerated, which can both increase and decrease in size, and are typically painless. The tumor in the present case was restricted to the base of the ear and no evidence of infiltration or metastasis was found. Progressive histiocytosis may spread and reach the lymphatic system through the lymph nodes, subsequently becoming systemic. The non-aggressive behavior observed in this case is possibly related to the non-epitheliotropic pattern. In the present case, MHC II histocompatibility complex markers, a phenotype compatible with dendritic cells, were used. Lysozyme antibodies marked histiocytic cells and the reactive lymphoid infiltrate was composed of CD20-positive B lymphoid lineage cells. Staining for E-cadherin was negative, negative results in labeling experiments is common, it is dependent upon the cellular origin of the leukocytes present in the sample. Staining for these molecules is recommended for differentiating feline progressive histiocytosis from Langerhans cells. Langerhans cells can be characterized by E-cadherin expression in about $10 \%$ of cases and marked Tymphocyte and neutrophil expression in the affected tissue. In this case, the histopathological exam along with immunohistochemistry was essential for differentiating them.
\end{abstract}

Keywords: tumor, histopathological, histocompatibility, histiocytic. 


\section{INTRODUCTION}

Histiocytic tumors in felines are nodules that commonly develop on limbs and head extremities. They can be divided into many subtypes including cutaneous histiocytoma [2,7], histiocytic sarcoma [5], reactive fibrohistiocytic nodule, Langerhans cell histiocytosis, and progressive feline dendritic cell histiocytosis $[1,4,6,8]$ Despite the same origin, they have behaviors that differ from each other, thus it is important to confirm diagnosis with histopathological and immunohistochemical tests, which can allow vets to differentiate between the different types, and early identification can facilitate prognosis and treatment [9].

In this study, we describe the pathological and immunohistochemical characteristics that can allow accurate diagnosis; enabling differentiation between non epitheliotropic progressive, histiocytosis of epitheliotropic and others feline neoplasms derived from histiocytes, such as histiocytomas, as well as common skin tumors, mastocytomas, and sarcomas.

\section{CASE}

A 5-year-old male, crossbreed, feline presented with a nodule at the base of the left auricle that was characterized by slow and progressive growth. Blood count and biochemistry were within the standards ranges considered normal for this species. The neoplasm of the ear region was circumscribed, and $1 \mathrm{~cm}$ in diameter and could be further described as a single, nodular, unattached, non-ulcerated, gelatinous mass, which was limited to the dermis layer (Figure 1). When an incision was made, the interior was soft and greasy in consistency. He was referred for histological processing performed with $\mathrm{HE}^{1}$.

Microscopy showed a dense, unencapsulated mass with circumscribed proliferation of histiocytic cells, which had abundant and eosinophilic cytoplasm (Figure 2A). The proliferative cells were enlarged and rounded, with centrally located nuclei ranging from round to oval in shape. These cells had broad cytoplasms, but sometimes in regions near the center of the nodule, the cytoplasm was scarce and with poorly defined edges. The cells infiltrated the tissues to the superficial dermis, and some cells, which had vacuoles were located at the extremities of the lesion. Some of these invading cells were associated with an inflammatory infiltrate consisting of lymphoplasmacytoid cells which intermingled with the neoplastic cells and limited cell proliferation (Figure 2B).
The neoplasm developed at the basement membrane without invasion of the underlying epidermis. Mitotic activity ranged from 3 to 4 mitoses per field in 40x magnification. CD20², E-cadherin ${ }^{2}$, $\mathrm{MHCII}^{2}$ (Figure 2C), and lysozyme ${ }^{2}$ (Figure 2D), antibodies were used for immunohistochemistry analysis.

\section{DISCUSSION}

The diagnosis of feline progressive histiocytosis in the present case was determined by histopathological and immunohistochemical evaluation. The histological type of the tumor was confirmed by observing that a proliferative lesion with a diffuse infiltration pattern of histiocytes, which did not reach the underlying dermis, was characteristic of the nonepitheliotropic type [6].

Progressive histiocytosis of feline dendritic cells, in both epitheliotropic and non-epitheliotropic forms, is considered a clinically progressive and rare disorder. There are reports which include cytological, clinical, histological and immunohistochemical examinations, but the diagnostic characteristics regarding the non-epitheliotropic classification have not yet

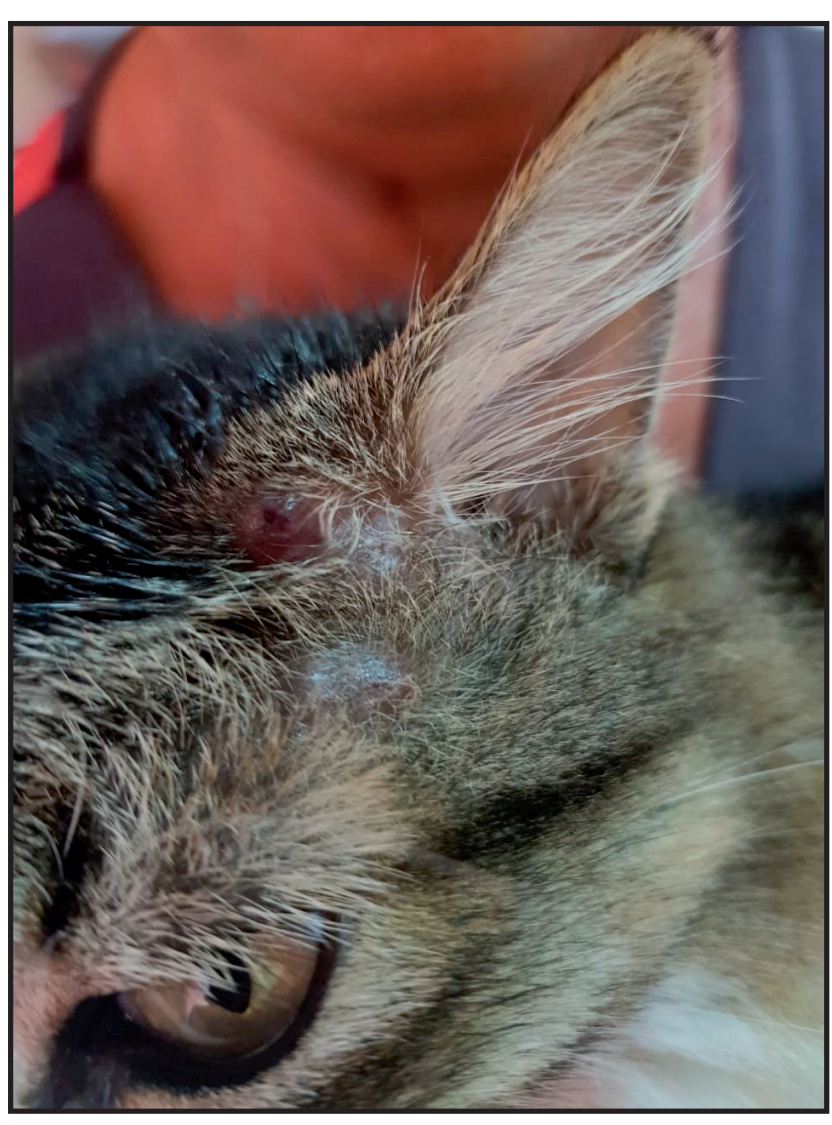

Figure 1. Progressive histiocytosis of non-epitheliotropic feline dendritic cells (HPD). Macroscopy of nodule at the base of the feline ear. Partly alopecic, rounded, and circumscribed. 


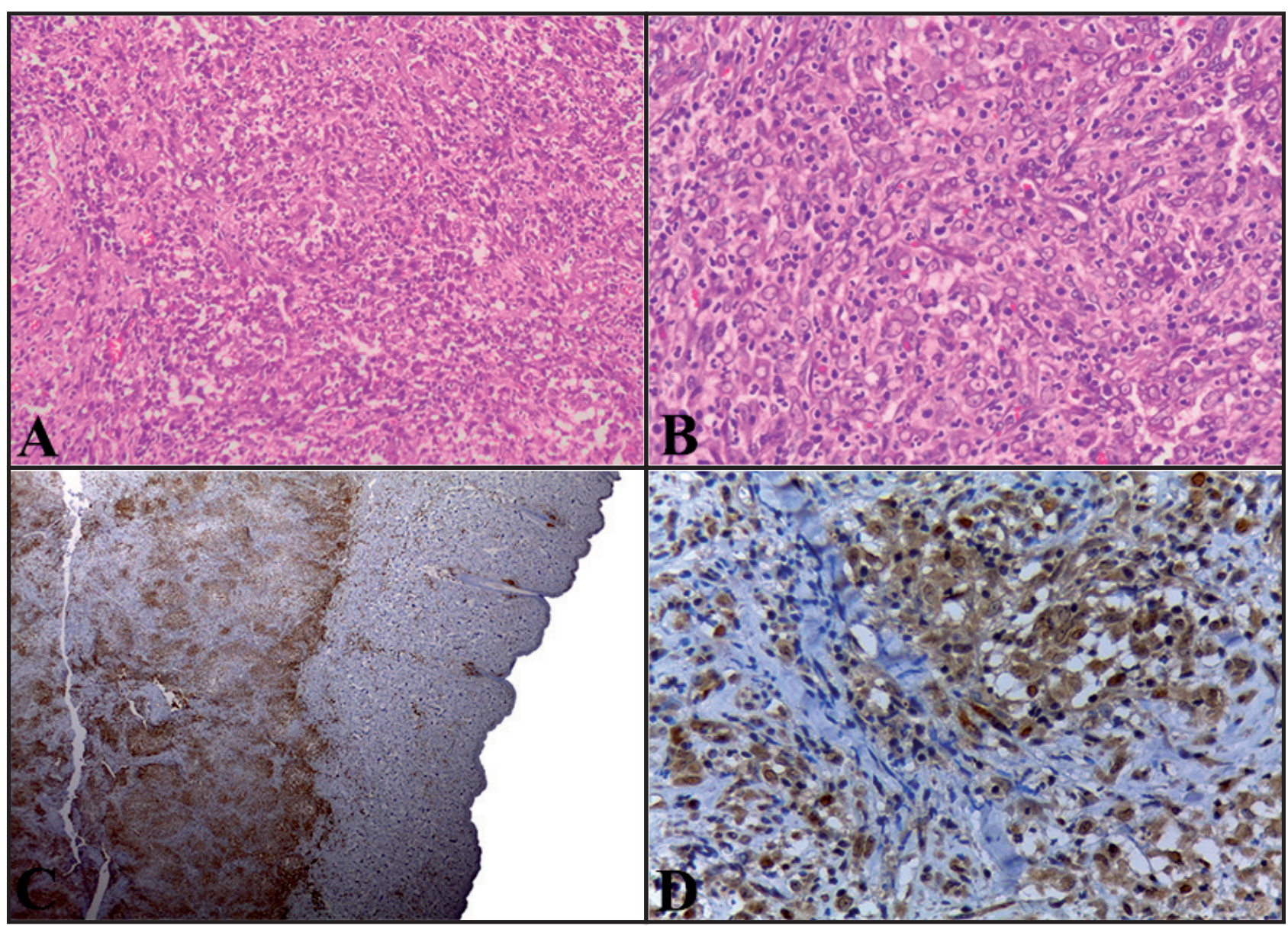

Figure 2. A- HPD microscopy showing circumscribed round cell proliferation, infiltration of randomly distributed plasma cells and lymphocytes, sometimes forming cords [HE; Magnification: 20x]. B- The histiocytes had proliferative and eosinophilic cytoplasm, proliferative, enlarged and rounded, with nucleus ranging from round to oval, centrally located [HE; Magnification: 40x]. C- HLA-DR antibody, highlighting cells of histiocytic origin densely labeled by the MHCII Histocompatibility complex. D- Intense brown marking of cells of histiocytic origin by the lysozyme antibody.

been properly identified [1]. Nodulations are predominantly observed in head and limb regions, usually non-ulcerated, which can both increase and decrease in size, and are typically painless $[6,9,11]$. There is no predisposition regarding age or breed, but a study involving 30 cases points to a greater predisposition among females [1].

The tumor in the present case was restricted to the base of the ear and no evidence of infiltration or metastasis was found. According to literature, progressive histiocytosis may spread and reach the lymphatic system through the lymph nodes, subsequently becoming systemic. When systemic, multiple nodules extend from the initial nodule and may become ulcerated and plaque-shaped [6-8,10]. The non-aggressive behavior observed in this case is possibly related to the non-epitheliotropic pattern.

In this study, the histological characteristics and immunohistochemistry both suggested the same diagnosis and were distinctly different to the characteristics typically seen in progressive histiocytosis types. In the microscopic examination, it was possible to observe that the tumor was restricted to the dermis layer, differing from the epitheliotropic type that often adhered to reaches epidermis and subcutaneous, but macroscopy could also become ulcerated and even form multiple masses or multinodular masses as the disease progressed [8].

This type of tumor is clinically indistinguishable from other neoplastic, allergic and inflammatory diseases, such as granulomas and tumor metastases [10], as well as other primary neoplasms, xanthomas, sarcomas and mastocytomas [5,6]. These conditions are important differentials within the histological examination and should be addressed initially by morphology and when possible, through specific markers in immunohistochemistry, as they commonly change over time [9].

For tumors of histiocytic origin antibodies should be applied, such as CD18, CD1, MHC II (major histocompatibility complex) that mark cells of histiocytic origin, while lysozyme antibodies can 
mark myeloid granulocytes and histiocytes $[3,6]$. In the present case, MHC II ${ }^{2}$ histocompatibility complex markers, a phenotype compatible with dendritic cells, were used. Lysozyme ${ }^{2}$ antibodies marked histiocytic cells and the reactive lymphoid infiltrate was composed of $\mathrm{CD} 20^{2}$-positive B lymphoid lineage cells. Staining for E-cadherin ${ }^{2}$ was negative, negative results in labeling experiments is common, it is dependent upon the cellular origin of the leukocytes present in the sample. Staining for these molecules is recommended for differentiating feline progressive histiocytosis from Langerhans cells [4].

Langerhans cells can be characterized by E-cadherin expression in about $10 \%$ of cases and marked $\mathrm{T}$ lymphocyte and neutrophil expression in the affected tissue. Approximately $30 \%$ of cases are cutaneous, while $10 \%$ of diagnosed cases are systemic. Importantly, the tumor can be successfully treated by corticosteroid, cyclosporine, and leflunomide-based medicines $[6,11]$. In this case, the histopathological exam along with immunohistochemistry was essential for differentiating them.

Improvements to the diagnosis of rare tumors are required. This study demonstrated the utility of various diagnostic methods, including clinical and histological characterization as well as immunohistochemical analysis, to confirm tentative diagnosis. Based on this report, it is possible to assist clinicians and pathologists regarding the diagnostic conduct.

\section{MANUFACTURERS}

${ }^{1}$ WCOR Corantes. São Paulo, SP, Brazil.

${ }^{2}$ Vetec Química Fina Ltda. Rio de Janeiro, RJ, Brazil.

Acknowledgements. We would like to thank all members of Hospital Veterinário of Universidade Federal da Paraíba. for their full support during this study. This work was supported by the CNPq (Brazil's National Research Council), [429862/2016-4].

Declaration of interest. The authors report no conflicts of interest. The authors alone are responsible for the content and writing of the paper.

\section{REFERENCES}

1 Affolter V.K. \& Moore P.F. 2006. Feline Progressive Histiocytosis. Veterinary Pathology. 43(5): 646-655.

2 Canelas M.M.C.A., Cardoso J.C.P.S., Andrade P.F., Reis J.P.G \& Tellechea O. 2010. Fibrous histiocytomas: histopathologic review of 95 cases. Anais Brasileiros de Dermatologia. 85: 211-215.

3 Cunha N.P., Ghisleni G., Scarampella F., Fabbrini F., Sforna M., Cornegliani L., Caniatti M., Avallone G., Moore P. \& Roccabianca P. 2014. Cytologic and immunocytochemical characterization of feline progressive histiocytosis. Veterinary Clinical Pathology. 43(3): 428-436.

4 Ferreira L.M., Emerich P.S., Diniz L.M., Lage L. \& Redighieri I. 2009. Langerhans cell histiocytosis: Letterer-Siwe disease - importance of dermatological diagnosis in two cases. Anais Brasileiros de Dermatologia. 84(4): 405-409.

5 Friedrichs K.R. \& Young K.M. 2008. Histiocytic sarcoma of macrophage origin in a cat: case report with a literature review of feline histiocytic malignancies and comparison with canine hemophagocytic histiocytic sarcoma. Veterinary Clinical Pathology. 37(1): 121-128.

6 Gross T.L., Ihrke P.J., Walder E.J. \& Affolter V.K. 2005. Epidermal Tumors. In: Skin diseases of the Dog and Cat: clinical and histopathologic diagnosis 2nd edn. Oxford: Blackwell Science, pp.829-833.

7 Mayr B., Wegscheider H., Loupal G. \& Reifinger M. 1996. Cytogenetic findings in two cases of feline histiocytoma. Journal of Small Animal Practice. 37(5): 239-240.

8 Pinto Da Cunha N., Ghisleni G., Scarampella F., Fabbrini F., Sforna M., Cornegliani L., Caniatti M., Avallone G., Moore P. \& Roccabianca P. 2014. Cytologic and immunocytochemical characterization of feline progressive histiocytosis. Veterinary Clinical Pathology. 43(3): 428-436.

9 Proença N.G. 2003. Histiocytes and non-Langerhans histiocytoses in Dermatology. Anais Brasileiros de Dermatologia. 78(1): 367-369.

10 Solc M., Jazic E., Crandell J. \& Loeffler D. 2017. Veterinary Record Case Reports. 5(2): e000428. doi: 10.1136/ vetreccr-2017-000428

11 Tizard I.R. 2014. Dendritic Cells and Antigen Processing. In: Veterinary Immunology 9.ed. Rio de Janeiro: Elsevier, 101p. 